\title{
Minimal component numbers of fixed point sets
}

\author{
by
}

Xuezhi Zhao (Beijing)

\begin{abstract}
Let $f:(X, A) \rightarrow(X, A)$ be a relative map of a pair of compact polyhedra. We introduce a new relative homotopy invariant $N^{\mathrm{C}}(f ; X, A)$, which is a lower bound for the component numbers of fixed point sets of the self-maps in the relative homotopy class of $f$. Some properties of $N^{\mathrm{C}}(f ; X, A)$ are given, which are very similar to those of the relative Nielsen number $N(f ; X, A)$.
\end{abstract}

1. Introduction. Let $f: X \rightarrow X$ be a self-map of a connected compact polyhedron $X$. The goal of Nielsen fixed point theory (see [1] or [3] for an introduction) is to understand the fixed point set $\operatorname{Fix}(f)=\{x \in X$ : $f(x)=x\}$ of $f$. An important invariant is the Nielsen number $N(f)$ of $f$, which is a lower bound for the number of fixed points of $f$ and also a lower bound for the component number of the fixed point set Fix $(f)$.

Relative Nielsen fixed point theory began with H. Schirmer's work ([5]), which is concerned with the information on the fixed point sets of maps of the form $f:(X, A) \rightarrow(X, A)$, called relative maps. The relative Nielsen number $N(f ; X, A)$ is a lower bound for the number of fixed points of a relative map $f:(X, A) \rightarrow(X, A)$, but it is not a lower bound for the component number of $\operatorname{Fix}(f)$. A simple example is the following:

Example 1.1. Let $f:(X, A) \rightarrow(X, A)$ be the identity map, where $X=I$ is the interval and $A$ consists of the two end points.

Recall from [5, Definition 2.4] that the relative Nielsen number $N(f ; X, A)$ is defined to be the sum of the Nielsen number $N(\bar{f})$ of the restriction $\bar{f}: A \rightarrow A$ of $f$ to $A$ and the number of essential fixed point classes of $f$ which do not contain any essential fixed point classes of $\bar{f}$. We have

2000 Mathematics Subject Classification: 55M20, 54H25.

This result was announced at a special session on fixed point theory of the geometric topology conference at Xi'an, a satellite conference of ICM 2002. That special session was dedicated to the memory of Helga Schirmer.

This project was supported by the Beijing Natural Sciences Foundation and National Natural Sciences Foundation of China. 
$N(f ; X, A)=N(\bar{f})+N(f)-N(f, \bar{f})=2+1-1=2$. But the component number of $\operatorname{Fix}(f)$ is 1 .

So, in order to estimate the component numbers of the fixed point sets of relative maps, one needs to seek new invariants other than the relative Nielsen number.

It is the purpose of this paper to give a relative homotopy invariant lower bound for the component numbers of the fixed point sets of relative self-maps.

In Section 2, we define a new Nielsen type number $N^{\mathrm{C}}(f ; X, A)$ and show that it is a lower bound for the component number of $\operatorname{Fix}(f)$. Some properties of $N^{\mathrm{C}}(f ; X, A)$ are also given. Section 3 is devoted to the proof of the minimum theorem for $N^{\mathrm{C}}(f ; X, A)$. In Section 4, we show the relations among the Nielsen number, minimal fixed point number and minimal component number of the fixed point set for maps $f: X \rightarrow X$ without an invariant subspace $A$.

2. A lower bound. Let us consider relative maps of the form $f:(X, A)$ $\rightarrow(X, A)$. The homotopies between such maps are also relative, i.e. of the form $H:(X \times I, A \times I) \rightarrow(X, A)$. Define

$\operatorname{MCF}[f ; X, A]:=\min \{$ component number of $\operatorname{Fix}(g)$ :

$$
g \text { is relatively homotopic to } f\} \text {. }
$$

In this section we shall give a lower bound for $\operatorname{MCF}[f ; X, A]$.

Definition 2.1. Let $f:(X, A) \rightarrow(X, A)$ be a relative map. We define $N^{\mathrm{C}}(f ; X, A)$ to be the sum of the number of essential fixed point classes of $f$ and the number of inessential fixed point classes of $f$ which contain essential fixed point classes of $\bar{f}$, i.e.

$$
N^{\mathrm{C}}(f ; X, A):=N(f)+\left|i_{\mathrm{FPC}}\left(\mathrm{FPC}_{\mathrm{e}}(\bar{f})\right) \cap \mathrm{FPC}_{\mathrm{i}}(f)\right|,
$$

where $|\cdot|$ denotes the cardinality of a set, $\mathrm{FPC}_{\mathrm{e}}(\cdot)$ and $\mathrm{FPC}_{\mathrm{i}}(\cdot)$ are the sets of essential and inessential fixed point classes respectively, and $i_{\mathrm{FPC}}: \operatorname{FPC}(\bar{f})$ $\rightarrow \mathrm{FPC}(f)$ is the natural inclusion (see [3, III, 1.8(ii)]) of the set of fixed point classes of $\bar{f}$ in the set of fixed point classes of $f$.

Recall from [5, Definition 2.1] that a fixed point point class of $f$ is said to be common if it contains an essential fixed point class of $\bar{f}$. So, $N^{\mathrm{C}}(f ; X, A)$ is the sum of $N(f)$ and the number of inessential common fixed point classes.

Using the basic relative Nielsen numbers from [6], we have

Proposition 2.2.

$$
\begin{aligned}
N^{\mathrm{C}}(f ; X, A)= & N(f)+N_{0101}(f ; X, A)+N_{0110}(f ; X, A) \\
& +N_{0111}(f ; X, A)+N_{0100}(f ; X, A) .
\end{aligned}
$$


From the homotopy invariance of the basic relative Nielsen numbers ( $[6$, Theorem 3.1]), we get immediately the homotopy invariance of $N^{\mathrm{C}}(f ; X, A)$. Similarly, we can show commutativity and homotopy type invariance of $N^{\mathrm{C}}(f ; X, A)$. We omit the details.

Theorem 2.3 (Lower bound theorem). Let $f:(X, A) \rightarrow(X, A)$ be a relative map. Then the component number of the fixed point set of any map relatively homotopic to $f$ is at least $N^{\mathrm{C}}(f ; X, A)$.

Proof. Each essential fixed point class of $f$ is a non-empty set. An inessential fixed point class of $f$ is also non-empty if it contains an essential fixed point class of $\bar{f}$. Note that fixed points in different fixed point classes of $f$ are in different components of $\operatorname{Fix}(f)$. Thus the component number of $\operatorname{Fix}(f)$ is greater than or equal to the sum of the number of essential fixed point classes of $f$ and the number of inessential fixed point classes of $f$ containing essential fixed point classes of $\bar{f}$, which is just $N(f)+$ $\left|i_{\mathrm{FPC}}\left(\mathrm{FPC}_{\mathrm{e}}(\bar{f})\right) \cap \mathrm{FPC}_{\mathrm{i}}(f)\right|=N^{\mathrm{C}}(f ; X, A)$.

Since $N^{\mathrm{C}}(f ; X, A)$ is a relative homotopy invariant, the conclusion holds for all maps relatively homotopic to $f$.

In the example of the first section, the unique non-empty fixed point class of $f$ is essential and contains all essential fixed point classes of $\bar{f}$. So, $N^{\mathrm{C}}(f ; X, A)=1$, which is equal to $\operatorname{MCF}[f ; X, A]$.

A relation among $N^{\mathrm{C}}(f ; X, A)$ and other Nielsen type numbers is:

Proposition 2.4. For any relative map $f:(X, A) \rightarrow(X, A)$, we have

$$
N(f) \leq N^{\mathrm{C}}(f ; X, A) \leq N(f ; X, A) .
$$

Proof. The first inequality is trivial. Since every fixed point class of $\bar{f}$ is contained in a unique fixed point class of $f$, the number $N(f, \bar{f})$ of essential fixed point classes of $f$ containing essential fixed point classes of $\bar{f}$ is less than or equal to the number of essential fixed point classes of $\bar{f}$ contained in essential fixed point classes of $f$. Thus,

$$
\begin{aligned}
N(f ; X, A) & =N(f)+N(\bar{f})-N(f, \bar{f}) \\
& \geq N(f)+N(\bar{f})-\left|\left\{\mathbf{F} \in \mathrm{FPC}_{\mathrm{e}}(\bar{f}): i_{\mathrm{FPC}}(\mathbf{F}) \in \mathrm{FPC}_{\mathrm{e}}(f)\right\}\right| \\
& =N(f)+\left|\left\{\mathbf{F} \in \mathrm{FPC}_{\mathrm{e}}(\bar{f}): i_{\mathrm{FPC}}(\mathbf{F}) \in \mathrm{FPC}_{\mathrm{i}}(f)\right\}\right| \\
& \geq N(f)+\left|i_{\mathrm{FPC}}\left(\mathrm{FPC}_{\mathrm{e}}(\bar{f})\right) \cap \mathrm{FPC}_{\mathrm{i}}(f)\right|=N^{\mathrm{C}}(f ; X, A) .
\end{aligned}
$$

The next example shows that the inequalities above can be strict.

ExAmple 2.5. Let $(X, A)=\left(S^{1}, S^{0}\right)$ and $f:(X, A) \rightarrow(X, A)$ be the identity map.

It is clear that $N(f)=0$ and $N(\bar{f})=2$. Since the two essential fixed point classes of $\bar{f}$ are contained in the inessential fixed point class of $f$ 
which is just the total fixed point set, we have $N^{\mathrm{C}}(f ; X, A)=0+1=1$. But $N(f ; X, A)=N(f)+N(\bar{f})-N(f, \bar{f})=0+2-0=2$.

On the other hand, we have

Proposition 2.6. For any relative map $f:(X, A) \rightarrow(X, A)$,

(1) $N(f)=N^{\mathrm{C}}(f ; X, A)$ if and only if each essential fixed point class of $\bar{f}: A \rightarrow A$ is contained in an essential fixed point class of $f$, i.e. there is no inessential common fixed point class.

(2) $N^{\mathrm{C}}(f ; X, A)=N(f ; X, A)$ if and only if each fixed point class of $f$ contains at most one essential fixed point class of $\bar{f}: A \rightarrow A$.

3. Minimum theorem. In this section, we show that under some assumptions on $(X, A)$, our new Nielsen type number $N^{\mathrm{C}}(f ; X, A)$ can be realized as the component number of the fixed point set of a map in the relative homotopy class of the given map $f:(X, A) \rightarrow(X, A)$.

Lemma 3.1. Let $(X, A)$ be a pair of compact polyhedra such that $X-A$ has no local cut point and is not a 2-manifold. Let $c: I \rightarrow X$ be a path in $X$ with $c((0,1)) \subset X-A$. Then $c$ is homotopic, with end points fixed, to a path $c^{\prime}$ such that $c^{\prime}((0,1)) \subset X-A$ and $c^{\prime}$ has no self-intersection points.

Proof. Since $X-A$ is not a 2-manifold, after a barycentric subdivision there is a 1 -simplex $\sigma$ in $X-A$ which is a face of at least three 2 -simplices. By a homotopy, we can make $c$ intersect $\sigma$ transversely.

Suppose that $c(\bar{t}) \in \sigma$. Write $S$ for the set of self-intersection points of $c$. By general position, the pre-image of $S$ is a finite set with $S \cap \sigma=\emptyset$. Write $c^{-1}(S)=\left\{t_{1}, \ldots, t_{n}\right\}$. Let $t_{i}$ be in $c^{-1}(S)$ so that $\left|t_{i}-\bar{t}\right|=\min _{1 \leq k \leq n}\left|t_{k}-\bar{t}\right|$.

The figure below shows that we can remove the intersection point $c\left(t_{i}\right)$ by a homotopy relative to $\left[0, t_{j}^{\prime}\right] \cup\left[t_{j}^{\prime \prime}, 1\right]$, where $\left(t_{j}^{\prime}, t_{j}^{\prime \prime}\right)$ is a small neighborhood of $t_{j}$ in the interval $I$ with $\left(t_{j}^{\prime}, t_{j}^{\prime \prime}\right) \cap c^{-1}(S)=\left\{t_{j}\right\}, c^{-1}\left(c\left(t_{i}\right)\right)=\left\{t_{i}, t_{j}\right\}$ and $t_{j} \neq t_{i}$.

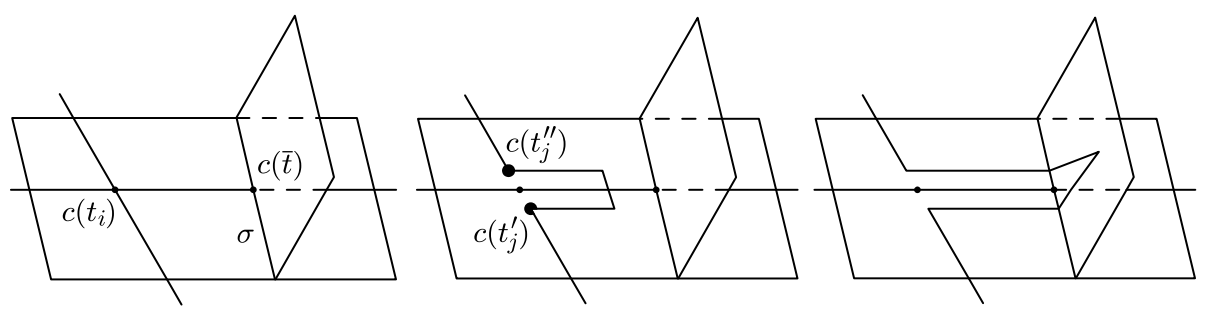

Repeating the above procedure, we get the desired path.

By using the same method, we get

Lemma 3.2. Let $(X, A)$ and a path $c$ satisfy the condition in Lemma 3.1. Suppose that $c$ has no self-intersection point, and that there is a 1-simplex 
$\sigma$ in $X-A$ which is a face of at least three 2-simplices such that $c$ intersects $\sigma$ transversely. If $d: I \rightarrow X$ is a path with $d((0,1)) \subset X-A$, then $d$ is homotopic, with end points fixed, to a path $d^{\prime}$ such that

1) $d^{\prime}(I) \cap c(I)=\emptyset$

2) $d^{\prime}((0,1)) \subset X-A$,

3) $d^{\prime}$ has no self-intersection points.

The idea of the proof of the above lemma is similar to those in [2, Lemmas 3.1, 3.2, 3.3]. But the end points of the paths here may be local cut points or lie in boundary simplices. So, the methods in [2] do not work directly in our case. Also, as in [2], the proof is concentrated on the case where $X-A$ is a 2-dimensional complex because the $c^{\prime}$ and $d^{\prime}$ will be obtained easily by general position if $X-A$ is high-dimensional.

Now we present the minimum theorem for the Nielsen type number $N^{\mathrm{C}}(f ; X, A)$. Similarly to the existing minimum theorems in relative Nielsen fixed point theory, we need the so-called by-pass condition:

Definition 3.3 ([5, Definition 5.1]). A subspace $A$ of a space $X$ is said to be by-passed if every path in $X$ with end points in $X-A$ is homotopic relative end points to a path in $X-A$.

Theorem 3.4. Let $(X, A)$ be a pair of compact polyhedra so that

(i) $X$ is connected,

(ii) $X-A$ has no local cut point and is not a 2-manifold,

(iii) every component of $A$ is a Nielsen space (i.e. each self-map $g: A \rightarrow A$ is homotopic to a map with $N(g)$ fixed points that can be located anywhere in $A$ (see [5, p. 466]),

(iv) $A$ can be by-passed in $X$.

Then any map $f:(X, A) \rightarrow(X, A)$ is relatively homotopic to a map $g:(X, A)$ $\rightarrow(X, A)$ such that the component number of the fixed point set of $g$ is $N^{\mathrm{C}}(f ; X, A)$.

Proof. Since $A$ is a Nielsen space up to a relative homotopy, we can assume that $f$ has $N(\bar{f})$ fixed points in $A$, which are all on the boundary $\operatorname{Bd}(A)$ of $A$ in the total space $X$.

Let $x$ and $y$ be any two fixed points in $A$ which are in the same fixed point class of $f$. By definition, there is a path $c_{x y}: I \rightarrow X$ from $x$ to $y$ such that $c_{x y} \simeq f\left(c_{x y}\right)$ rel $\{0,1\}$. Such a path is said to be a Nielsen path connecting $x$ and $y$. As $A$ can be by-passed in $X$, the Nielsen path $c_{x y}$ can be chosen so that $c_{x y}((0,1)) \subset X-A$. Since $X-A$ has no local cut point and is not a 2-manifold, by Lemma 3.1 we can assume that $c_{x y}$ has no self-intersection and intersects transversely a 1 -simplex $\sigma$ in $X-A$, which is a face of at least three 2-simplices. 
Repeating the procedure for each pair of fixed points in $A$ which are in the same fixed point class of $f$, we get all possible Nielsen paths for the fixed points in $A$. By Lemma 3.2, we can homotope these paths so that they are pairwise disjoint except for end points and have no self-intersections, and that each path has at least one transversal intersection point with the simplex $\sigma$.

Write $Y=\bigcup_{x, y} c_{x y}(I)$ for the union of all the Nielsen paths. Thus, $\left.f\right|_{Y}: Y \rightarrow X$ is homotopic to the inclusion $i: Y \rightarrow X$. By the homotopy extension property, $f$ is homotopic to a map $f^{\prime}:(X, A) \rightarrow(X, A)$ such that $f^{\prime}(a)=f(a)$ for all $a \in A$ and $f^{\prime}(y)=y$ for all $y \in Y$. Because each point on a Nielsen path is changed into a fixed point, the fixed points in $A$ lying in the same fixed point classes of $f^{\prime}$ on $X$ are in the same connected components of the fixed point set of $f^{\prime}$.

Let $e$ be a path in $X$ with end points in $X-(Y \cup A)$. Since $A$ can be by-passed in $X$, there is a path $e^{\prime}$ in $X-A$ such that $e \simeq e^{\prime} \operatorname{rel}\{0,1\}$. By Lemma 3.2, we can remove the intersection points $e^{\prime}(I) \cap Y$. So, $e^{\prime}$ is homotopic to a path in $X-(Y \cup A)$. It follows that $Y \cup A$ can be bypassed in $X$. Applying the method of the proof of [5, Theorem 6.2] to the relative map $f^{\prime}:(X, Y \cup A) \rightarrow(X, Y \cup A)$, we can unite the fixed points in $X-Y \cup A$ which are in the same fixed point class, and combine the fixed points to $\operatorname{Bd}(A)$ if they lie in common fixed point classes, i.e. they contain fixed points on $A$. Finally, deleting the fixed points in $X-Y \cup A$ of index zero, we get the desired map $g:(X, A) \rightarrow(X, A)$.

The assumption in the theorem above is the same as in H. Schirmer's minimum theorem ([5, Theorem 6.2]). Under this assumption, local cut points of $X$ may be located on $A$, especially on $\operatorname{Bd}(A)$. But a local cut point of $X$ lying on $\operatorname{Bd}(A)$ is not an obstruction to combining the fixed points in $X-A$ to $\operatorname{Bd}(A)$, because we have

Proposition 3.5. Let $(X, A)$ be a pair of compact polyhedra. If $A$ can be by-passed in $X$ and there is a local cut point a of $X$ lying on $\operatorname{Bd}(A)$, then the component of $A$ containing a is simply connected and its boundary is just $\{a\}$.

4. Case of ordinary maps. In this final section, we make some comments about estimating the component number of the fixed point sets of ordinary self-maps, i.e. maps without an invariant subspace.

Let $f: X \rightarrow X$ be a self-map of a compact polyhedron $X$. As in the relative case, one may wish to estimate the component number of $\operatorname{Fix}(f)$. Set

$\mathrm{MCF}[f]=\min \{$ component number of $\operatorname{Fix}(g): g \simeq f\}$. 
It is well known that the Nielsen number $N(f)$ of $f$ is a lower bound for the number of fixed points of $f$. Noticing that fixed points in the same component of the fixed point set are in the same fixed point class, we have

Proposition 4.1. Let $f: X \rightarrow X$. Then $N(f) \leq \operatorname{MCF}[f] \leq \operatorname{MF}[f]$.

Corollary 4.2. For the identity map id: $X \rightarrow X$, we have

$$
\mathrm{MCF}[\mathrm{id}]= \begin{cases}1 & \text { if } \mathrm{MF}[\mathrm{id}]>0 \\ 0 & \text { if } \mathrm{MF}[\mathrm{id}]=0\end{cases}
$$

The following example shows that the inequalities in the above proposition can be strict.

ExAmPle 4.3. Let $K$ be the following graph:

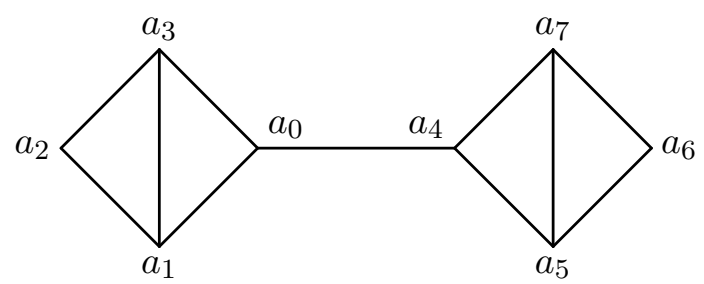

If we attach 33 copies of $S^{2}$ along the edge $\left[a_{0}, a_{4}\right]$ and attach a disc with three holes along each edge of $K$ except $\left[a_{0}, a_{4}\right]$, we get a 2-dimensional complex $L$. Let $f: L \rightarrow L$ be the identity.

Clearly, $L$ is connected, hence $\operatorname{dim} H_{0}(K)=1$. Since $\operatorname{dim} H_{1}(K)=4$, after attaching the 10 discs with three holes, we have $\operatorname{dim} H_{1}(L)=4+3 \cdot 10=$ 34. As $\operatorname{dim} H_{2}(K)=33$, the Euler characteristic $\chi(L)$ is $1-34+33=0$. We have $N(f)=0$.

Note that the connected complex $L$ has eleven 2-dimensional branches (see [4, p. 91] for definition). Since the Euler characteristic of each 2-dimensional branch is either 34 or $-2, L$ is therefore a special case of $K_{4}$ in $[4$, IV Example 3.3]. By [4, p. 95], we have $\mathrm{MF}[f]=5$. By Corollary 4.2, we have $\mathrm{MCF}[f]=1$.

Thus, for this map $f$, we have $N(f)<\operatorname{MCF}[f]<\operatorname{MF}[f]$.

\section{References}

[1] R. F. Brown, The Lefschetz Fixed Point Theorem, Scott, Foresman and Co., Glenview, IL, 1971.

[2] B. Jiang, On the least number of fixed points, Amer. J. Math. 102 (1980), 749-763.

[3] - Lectures on Nielsen Fixed Point Theory, Contemp. Math. 14, Amer. Math. Soc., Providence, RI, 1983.

[4] T. H. Kiang, The Theory of Fixed Point Classes, Springer, Berlin, 1989. 
[5] H. Schirmer, A relative Nielsen number, Pacific J. Math. 122 (1986), 459-473.

[6] X. Z. Zhao, Basic relative Nielsen numbers, in: Topology-Hawaii, World Sci., 1992, $215-222$.

Department of Mathematics

Capital Normal University

Beijing 100037, P.R. China

E-mail: zhaoxve@mail.cnu.edu.cn

Received 6 January 2003;

in revised form 18 August 2003 